\title{
On the Performance of Cognitive Underlay SIMO Networks over Equally Correlated Rayleigh Fading Channels
}

\author{
Tu Lam Thanh, Vo Nguyen Quoc Bao, Pham Thi Dan Ngoc, Tran Trung Duy \\ Posts and Telecommunications Institute of Technology, Ho Chi Minh City, Vietnam \\ Correspondence: Vo Nguyen Quoc Bao, baovnq@ptithcm.edu.vn \\ Manuscript communication: received 11 February 2015, accepted 13 July 2015
}

\begin{abstract}
The performance of single-input multiple-output (SIMO) cognitive spectrum sharing networks with the presence of equally correlated Rayleigh fading channels is investigated. In particular, based on the truncated infinitive series of cumulative distribution function (CDF) and probability density function (PDF) of the end-to-end signal-to-noise ratios (SNRs), close-form expressions are provided for the system outage performance, bit error rate and ergodic capacity. It is shown that the system performance merely depends on the correlation coefficient between antennas. Monte-Carlo simulations are also contributed to confirm the accuracy of our analysis.
\end{abstract}

Keywords- MIMO, underlay cognitive radio network, equally correlated Rayleigh fading, bit error rate, ergodic capacity.

\section{INTRODUCTION}

In wireless communications, there is a huge demand for radio spectrum resources due to the rapid growth of mobile applications and users. In addition, the inefficient utilization of frequency bands forces researchers as well as regulatory authorities to promote new spectrum access approach called dynamic spectrum sharing (DSA) or cognitive radio (CR) [1-3]. The concept of DSA/CR can be used not only to deploy next generation wireless systems on the allocated spectrum but also to improve the capacity of currently existing wireless systems.

In the conventional spectrum sharing model, unlicensed users (or secondary users SUs) utilize the licensed bands, which are temporarily unused by primary users (PU). Such a protocol is harshly implemented in practice since its performance significantly depends on available spectrum bands left by PUs or PUs activities. Recently, underlay spectrum sharing protocols have been proposed and studied in [4-8], where the SUs can operate concurrently with primary users as long as their transmissions do not deteriorate the quality of service (QoS) of PU.

On the other hand, multiple-input multiple-output (MIMO) is an advanced technology that can effectively exploit spatial multiplexing and antenna diversity gains as compared with traditional single-input single output (SISO) systems $[9,10]$. However, the performance of multi-antenna systems is often severely degraded due to the correlation effect between adjacent antennas, (e.g., see [11-13]). In particular, the authors in [11] studied the outage probability (OP) of dual hop equally correlated Rayleigh fading channels. It is shown that the diversity order is equal to the number of available re- lays regardless of the correlation coefficient values. Taking into account the effect of co-channel interference, Sekulovic et al. investigated the outage performance of single hop selection combiner using exponential correlation model [12]. In [13], the performance for partial relay selection of a dual-hop relay system with correlated antennas at each node was studied, where orthogonal space time block coding is employed at the source and the best relay.

Recently, MIMO technology has been considered for cognitive radio networks, (e.g., see [14-17]). In particular, orthogonal transmissions for spectrum underlay MISO cognitive radio were considered in [14]. In [15], the performance of cognitive underlay Alamouti schemes was investigated over Rayleigh fading channels. Paper [16] proposed antenna selection (AS)based multiple antenna techniques for interferenceconstrained underlay cognitive radio networks to exploit spatial diversity with less hardware and to improve secondary system performance. Numerical results show that the optimal transmit rule is a nonlinear function of the power gain of the channel from the secondary transmit antenna to the primary receiver and from the secondary transmit antenna to the secondary receive antenna. In [17], the achievable spatial throughput of a multi-antenna Poisson cognitive radio network collocated with a Poisson multi-antenna primary network was investigated. It is also shown that there exists an optimal medium access probability which maximizes the system spatial throughput.

However, all of the above-mentioned works have focused on the impact of correlated fading channels in un-cognitive systems. In this paper, we for the first time investigate underlay SIMO networks over equally correlated Rayleigh fading channels. 


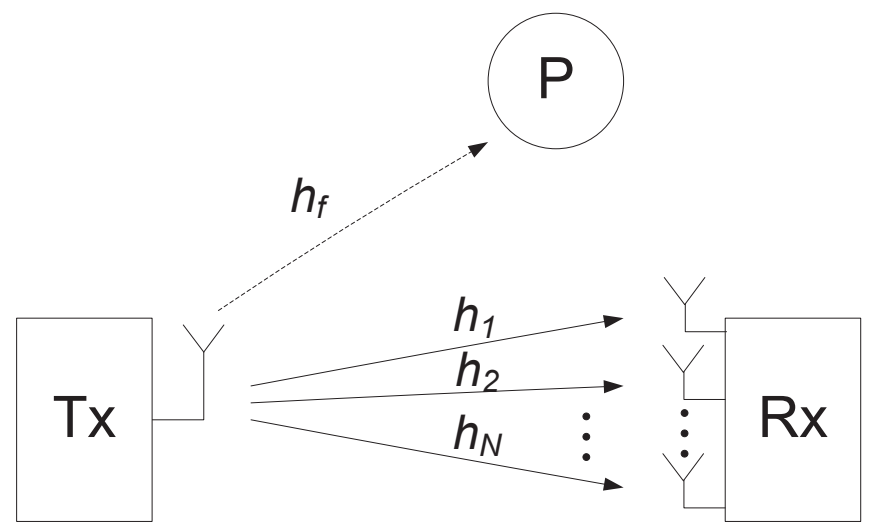

Figure 1. Cognitive radio over correlated Rayleigh fading.

Our main contribution in this paper is summarized as follows:

- We develop a system model and a corresponding derivation framework to take into account the correlation effect not only from adjacent antennas but also from the interference link between the secondary transmitter to the primary receiver.

- We obtain exact closed-form expression for outage probability, average bit error rate and ergodic capacity, which are then used to derive their bounds for efficient computation.

- We investigate effects of the primary user's position as well as the correlation effect of adjacent receive antennas.

The rest of this paper is organized as follows. In Section 2, the system model under consideration is presented. Using the probability density function (PDF)based approach, the exact and asymptotic performance analyses in terms of outage probability, average bit error rate and ergodic capacity over equally correlated Rayleigh fading channels are presented in Section 3. In Section 4, we provide some typical numerical results to verify the analysis. Finally, we conclude this paper in Section 5 .

\section{System Model}

The system under consideration is illustrated in Figure 1 , where the secondary network operates on the same frequency band licensed to the primary network $(\mathrm{P})$. The secondary network consists of one singleantenna transmitter (Tx) and one receiver $(\mathrm{Rx})$, which is equipped with $\mathcal{N}$ antennas ${ }^{1}$.

Let us denote $h_{l}$ with $l \in\{1, \ldots, \mathcal{N}\}$ as the channel coefficient of the channel from Tx to the $l$-th antenna of Rx. Taking into account correlation effect among adjacent antennas, $h_{l}$ can be modeled by making use the approach in $[19,20]$ as follows:

$$
h_{l}=\sqrt{1-\rho} X_{l}+\sqrt{\rho} X_{0}+j\left(\sqrt{1-\rho} Y_{l}+\sqrt{\rho} Y_{0}\right),
$$

where $j=\sqrt{-1}$. Furthermore, $X_{0}, Y_{0}, X_{l}$, and $Y_{l}$ are the set of normal distribution RVs with zero mean and

\footnotetext{
${ }^{1}$ In this paper, we extend our previous work in [18], which limits on two antennas.
}

variance $\lambda / 2$ [19]. Here, $\rho$ denotes the cross correlation coefficient between $h_{l}$ and $h_{p}(l \neq p)$, given by

$$
\rho=\frac{E\left\{h_{l} h_{p}^{*}\right\}}{\sqrt{E\left\{\left|h_{l}\right|^{2}\right\} E\left\{\left|h_{p}^{*}\right|^{2}\right\}}}, \quad 0 \leq \rho \leq 1 .
$$

In (2), $E\{X\}$ and $X^{*}$ respectively denote the average and the complex conjugate of $X$. Conditioned on $u=X_{0}^{2}+Y_{0}^{2}$, the channel gain, $\left|h_{l}\right|^{2}$, will follow non central chi-square distribution with two degrees of freedom and non-centrality parameter $\rho$ u, i.e., $\chi_{2}\left(\sqrt{\rho u}, \frac{(1-\rho) \lambda}{2}\right)[20]$. As a result, the conditional CDF of $\left|h_{l}\right|^{2}$ is given by [21]

$$
F_{\left|h_{l}\right|^{2} \mid u}(z \mid u)=1-Q\left(\sqrt{\frac{2 \rho u}{(1-\rho) \lambda}}, \sqrt{\frac{2 z}{(1-\rho) \lambda}}\right),
$$

where $Q(.,$.$) is Marcum-Q function [22, Eq. 1].$

For the underlay approach, the transmit power of secondary networks are constrained by the maximum tolerable interference power at the primary receiver, $\mathcal{I}_{p}$. Mathematically, we have [23]

$$
P=\frac{\mathcal{I}_{p}}{\left|h_{f}\right|^{2}}
$$

where $h_{f}$ denotes the channel coefficient of the interference link from $\mathrm{Tx}$ to the primary receiver. For Rayleigh fading, $\left|h_{f}\right|^{2}$ is exponentially distributed with parameter $\omega$. Although optimum performance of maximal ratio combining is highly desirable, practical wireless systems often sacrifice some performance in order to reduce their complexity, i.e., hardware-feasible and cheaper to implement. In this paper, we adopt selection combiner as the receiver diversity combiner, which simply requires SNR measurements. With selection combining, $\mathrm{Rx}$ will select the link having the largest instantaneous SNR for decoding. As a result, the endto-end SNR at the output of selective combiner is given as [24]

$$
\gamma_{\Sigma}=\max \left(\gamma_{1}, \gamma_{2}, \ldots, \gamma_{\mathcal{N}}\right)
$$

where $\gamma_{l}=P\left|h_{l}\right|^{2} / \mathcal{N}_{0}$ with $\mathcal{N}_{0}$ being the noise variance.

\section{Performance Analysis}

In this section, we will investigate the performance of cognitive underlay SIMO systems over equally correlated Rayleigh fading channels. Starting from the endto-end SNR of the system under consideration, we first derive the cumulative distribution function (CDF) and the PDF, which are then used to obtain important system performance metrics including outage probability, bit error rate for $\mathcal{M Q A M}$ and Shannon capacity.

\subsection{The Cumulative Distribution Function (CDF)}

Making use the fact that $\gamma_{l}$ with $l=1,2, \ldots, \mathcal{N}$ are not independent each other due to not only the interference link, i.e., $T=\left|h_{f}\right|^{2}$, but also the correlation effect 
among adjacent receiving antennas, i.e., $U=X_{0}^{2}+Y_{0}^{2}$, we can obtain the system CDF thanks to the conditional probability theorem. In particular, we have

$$
\begin{aligned}
F_{\gamma_{\Sigma}}(\gamma)= & \int_{0}^{\infty} \int_{0}^{\infty} F_{\gamma_{\Sigma} \mid(U, T)}(\gamma \mid(u, t)) f_{U}(u) f_{T}(t) d u d t \\
= & \int_{0}^{\infty} \int_{0}^{\infty}\left[1-Q\left(\sqrt{\frac{2 \rho u}{(1-\rho) \lambda}}, \sqrt{\frac{2 \gamma t \mathcal{N}_{0}}{(1-\rho) \mathcal{I}_{p} \lambda}}\right)\right]^{\mathcal{N}} \\
& \times \frac{1}{\omega} \exp \left(-\frac{t}{\omega}\right) \frac{1}{\lambda} \exp \left(-\frac{u}{\lambda}\right) d u d t
\end{aligned}
$$

It is important to note that the closed-form expression of $F_{\gamma_{\Sigma}}(\gamma)$ has not appeared in the literature except for the case of $\mathcal{N}=2$, which has recently reported in [18]. Since it is extremely difficult to analyze the exact closed-form expression for (6), we instead deploy the approximation approach. Starting from the series form of the first-order Marcum-Q function [25, Eq. 18], i.e.,

$$
Q(a, b)=\exp \left(-\frac{a^{2}}{2}\right) \sum_{k=0}^{\infty} \frac{\Gamma\left(k+1, \frac{b^{2}}{2}\right)}{k ! \Gamma(k+1)}\left(\frac{a^{2}}{2}\right)^{k}
$$

where $\Gamma(a, b)$ is upper incomplete gamma function [26, Eq. 8.350.2] and making use the binomial theorem, we can have the CDF of $\gamma_{\Sigma}$ after substituting (7) into (6) as

$$
\begin{aligned}
F_{\gamma_{\Sigma}}(\gamma) & =1+\sum_{n=1}^{\mathcal{N}} \sum_{k_{1}, k_{2}, \ldots, k_{n}=0}^{\infty}\left(\begin{array}{c}
\mathcal{N} \\
n
\end{array}\right)(-1)^{n}\left\{\int_{0}^{\infty}\right. \\
& \times\left(\frac{\rho}{(1-\rho) \lambda}\right)^{\sum_{i=1}^{n} k_{i}}\left[\prod_{i=1}^{n} \frac{\Gamma\left(k_{i}+1, \frac{\gamma t \mathcal{N}_{0}}{(1-\rho) \mathcal{I}_{p} \lambda}\right)}{k_{i} ! \Gamma\left(k_{i}+1\right)}\right] \\
& \times\left[\int_{0}^{\infty}(u)^{\sum_{i=1}^{n} k_{i}} \exp \left(-\frac{n \rho u}{(1-\rho) \lambda}\right) \frac{1}{\lambda} \exp \left(-\frac{u}{\lambda}\right) d u\right] \\
& \left.\times \frac{1}{\omega} \exp \left(-\frac{t}{\omega}\right) d t\right\}
\end{aligned}
$$

Using the result [26, Eq. 3.351.3] and [26, Eq, 8.352.2] and after tedious manipulations, we can get $F_{\gamma_{\Sigma}}(\gamma)$ as (9) shown at the top of the next page, where $\bar{\sum}, \alpha, \beta_{1, n}$, and $\beta_{2, n}$ are respectively defined as

$$
\begin{aligned}
\sum & =\sum_{m_{1}=0}^{k_{1}} \sum_{m_{2}=0}^{k_{2}} \cdots \sum_{m_{n}=0}^{k_{n}}, \\
\alpha & =\frac{\lambda(1-\rho)^{2} \mathcal{I}_{p}}{\omega \rho \mathcal{N}_{0}}, \\
\beta_{1, n} & =\frac{\rho}{(1-\rho)+n \rho}, \\
\beta_{2, n} & =\frac{\lambda(1-\rho) \mathcal{I}_{p}}{\omega n \mathcal{N}_{0}} .
\end{aligned}
$$

\subsection{The Probability Density Function (PDF)}

The PDF of $\gamma_{\Sigma}$ can be straightforwardly obtained from the first derivative of $F_{\gamma_{\Sigma}}(\gamma)$. In particular, dif- ferentiating (9) with respect to $\gamma$, we get

$$
\begin{aligned}
f_{\gamma_{\Sigma}}(\gamma) & =\frac{d F_{\gamma_{\Sigma}}(\gamma)}{d \gamma}=\alpha \sum_{n=1}^{\mathcal{N}} \sum_{k_{1}, k_{2}, \ldots, k_{n}=0}^{\infty} \bar{\sum}\left(\begin{array}{c}
\mathcal{N} \\
n
\end{array}\right) \\
& \times \frac{(-1)^{n}\left(\sum_{i=1}^{n} m_{i}\right) !\left(\sum_{i=1}^{n} k_{i}\right) !\left(\beta_{1, n}\right)^{1+\sum_{i=1}^{n} k_{i}}}{\prod_{i=1}^{n}\left[k_{i} ! m_{i} !\right](n)^{1+\sum_{i=1}^{n} m_{i}}} \mathcal{G},
\end{aligned}
$$

where $\mathcal{G}$ is given as

$$
\mathcal{G}= \begin{cases}-\frac{1}{\left(\gamma+\beta_{2, n}\right)^{2}}, & \text { if } \sum_{i=1}^{n} m_{i}=0 \\ \frac{\left(\sum_{i=1}^{n} m_{i}\right) \gamma^{\sum_{i=1}^{n} m_{i}-1}}{\left(\gamma+\beta_{2, n}\right)^{i=1} m_{i} m_{i}+1}-\frac{\left(\sum_{i=1}^{n} m_{i}+1\right) \gamma^{\sum_{i=1}^{n} m_{i}}}{\left(\gamma+\beta_{2, n}\right)^{\sum_{i=1}^{n} m_{i}+2}}, & \text { if } \sum_{i=1}^{n} m_{i} \neq 0 .\end{cases}
$$

\subsection{Outage Probability (OP)}

Outage probability is defined as the probability that the end-to-end SNR falls below a certain predetermined threshold SNR, $\gamma_{\text {th }}$. Mathematically, we have

$$
\mathrm{OP}=\operatorname{Pr}\left(\gamma_{\sum} \leq \gamma_{\mathrm{th}}\right)
$$

Having the CDF of $F_{\gamma_{\Sigma}}\left(\gamma_{\mathrm{th}}\right)$ at hands, one can get the closed-form expression for OP from (9) by replacing $\gamma$ by $\gamma_{\text {th }}$, i.e., OP $=F_{\gamma_{\Sigma}}\left(\gamma_{\text {th }}\right)$ as (14) shown at the top of the next page.

\subsection{Bit Error Rate (BER)}

Consider transmission of an $\mathcal{M}$-QAM signal over equally correlated Rayleigh fading channels, the average BER for cognitive underlay SIMO systems can be obtained by averaging its conditional BER in additive white Gaussian noise over the PDF of end-to-end SNR, that is, [27]

$$
\mathrm{BER}=\sum_{r=0}^{\log _{2} \sqrt{\mathcal{M}}} \sum_{c=0}^{v_{r}} \phi_{c}^{r} \int_{0}^{\infty} \operatorname{erfc}\left(\sqrt{\vartheta_{c} \gamma}\right) f_{\gamma_{\Sigma}}(\gamma) d \gamma,
$$

where $v_{r}=\left(1-2^{-r}\right) \sqrt{\mathcal{M}}-1, \vartheta_{c}=\frac{3(2 c+1)^{2} \log _{2} \mathcal{M}}{(2 \mathcal{M}-2)}$, and $\phi_{c}^{r}=\frac{(-1)^{\left\lfloor\frac{c 2^{r-1}}{\sqrt{\mathcal{M}}}\right\rfloor}\left(2^{r-1}-\left\lfloor\frac{c 2^{r-1}}{\sqrt{\mathcal{M}}}+\frac{1}{2}\right\rfloor\right)}{\sqrt{\mathcal{M}} \log _{2} \sqrt{\mathcal{M}}}$. Note that $\lfloor$.$\rfloor denotes$ the floor function. The closed-form expression for the average BER is provided in the following lemma.

Lemma 1 The system average BER for MQAM over equally correlated Rayleigh fading channels is of the form

$$
\begin{aligned}
\mathrm{BER}= & \alpha \sum_{r=0}^{\log _{2} \sqrt{\mathcal{M}}} \sum_{c=0}^{v_{r}} \sum_{n=1}^{\mathcal{N}} \sum_{k_{1}, k_{2}, \ldots, k_{n}=0}^{\infty} \bar{\sum}\left(\begin{array}{c}
\mathcal{N} \\
n
\end{array}\right)(-1)^{n} \\
& \times\left[\frac{\phi_{c}^{r}\left(\sum_{i=1}^{n} k_{i}\right) !\left(\sum_{i=1}^{n} m_{i}\right) !\left(\beta_{1, n}\right)^{1+\sum_{i=1}^{n} k_{i}}}{\prod_{i=1}^{n}\left[k_{i} ! m_{i} !\right](n)^{1+\sum_{i=1}^{n} m_{i}}}\right] \mathcal{D},
\end{aligned}
$$




$$
F_{\gamma_{\Sigma}}(\gamma)=1+\alpha \sum_{n=1}^{\mathcal{N}} \sum_{k_{1}, k_{2}, \ldots, k_{n}=0}^{\infty} \sum\left(\begin{array}{c}
\mathcal{N} \\
n
\end{array}\right)(-1)^{n}\left[\frac{\left(\sum_{i=1}^{n} k_{i}\right) !\left(\sum_{i=1}^{n} m_{i}\right) !}{\prod_{i=1}^{n}\left[k_{i} ! m_{i} !\right]}\right] \frac{\left(\beta_{1, n}\right)^{1+\sum_{i=1}^{n} k_{i}}}{(n)^{1+\sum_{i=1}^{n} m_{i}}}\left[\frac{1}{\gamma}\left(\frac{\gamma}{\gamma+\beta_{2, n}}\right)^{1+\sum_{i=1}^{n} m_{i}}\right]
$$

$$
\mathrm{OP}=1+\alpha \sum_{n=1}^{\mathcal{N}} \sum_{k_{1}, k_{2}, \ldots, k_{n}=0}^{\infty} \bar{\sum}\left(\begin{array}{c}
\mathcal{N} \\
n
\end{array}\right)(-1)^{n}\left[\frac{\left(\sum_{i=1}^{n} k_{i}\right) !\left(\sum_{i=1}^{n} m_{i}\right) !}{\prod_{i=1}^{n}\left[k_{i} ! m_{i} !\right]}\right] \frac{\left(\beta_{1, n}\right)^{1+\sum_{i=1}^{n} k_{i}}}{(n)^{1+\sum_{i=1}^{n} m_{i}}}\left[\frac{1}{\gamma_{\mathrm{th}}}\left(\frac{\gamma_{\mathrm{th}}}{\gamma_{\mathrm{th}}+\beta_{2, n}}\right)^{1+\sum_{i=1}^{n} m_{i}}\right]
$$

where $\mathcal{D}$ is given by

$$
\begin{aligned}
\mathcal{D}= & \left(\sum_{i=1}^{n} m_{i}\right) J_{1}\left(\vartheta_{\mathcal{c}}, \beta_{2, n}, \sum_{i=1}^{n} m_{i}, \sum_{i=1}^{n} m_{i}+1\right) \\
& -\left(\sum_{i=1}^{n} m_{i}+1\right) J_{1}\left(\vartheta_{\mathcal{c}}, \beta_{2, n}, \sum_{i=1}^{n} m_{i}+1, \sum_{i=1}^{n} m_{i}+2\right),
\end{aligned}
$$

and $\mathcal{J}_{1}$ is defined as

$$
J_{1}(a, b, n, m)=\frac{1}{\sqrt{\pi} \Gamma(m) b^{m-n}} G_{2,3}^{3,1}\left[\begin{array}{l|c}
a b & 0,1 \\
m-1,0, \frac{1}{2}
\end{array}\right] .
$$

Proof: With the help of [28], we begin the proof by representing $\operatorname{erfc}($.$) function in terms of Meijer-G$ function [26, Eq. 9.31.1] as follows:

$$
\operatorname{erfc}(\sqrt{x})=\frac{1}{\sqrt{\pi}} G_{1,2}^{2,0}\left[\begin{array}{c|c}
1 \\
0, \frac{1}{2}
\end{array}\right] \text {. }
$$

Plugging (19) and (11) into (15) and then taking the integral, i.e.,

$$
J_{1}(a, b, n, m)=\int_{0}^{\infty} \frac{\gamma^{n-1}}{(\gamma+b)^{m}} \operatorname{erfc}(\sqrt{a \gamma}) d \gamma
$$

yields the desired result as (16) [29].

\subsection{Ergodic Capacity}

The ergodic capacity for cognitive underlay SIMO networks over equally correlated Rayleigh fading channels can be obtained by averaging the capacity of an additive white Gaussian noise over the distribution of the end-to-end system SNR. Mathematically, we have

$$
C=B \int_{0}^{\infty} \log _{2}\left(1+\gamma_{\Sigma}\right) f_{\gamma_{\Sigma}}(\gamma) d \gamma
$$

where $B$ is the channel bandwidth in Hz. The following Proposition is particularly useful in finding the system ergodic capacity.

Proposition 2 For integer numbers $n$ and $m$ with $m>n$ and $b>0$, we have

$$
\begin{aligned}
J_{2}(b, n, m) & =\int_{0}^{\infty} \frac{x^{n-1}}{(x+b)^{m}} \log _{2}(1+x) d x \\
& =\frac{(b)^{n-m}}{\log (2)(m-1) !} G_{3,3}^{2,3}\left(b \mid \begin{array}{c}
1,1,1-n \\
1, m-n, 0
\end{array}\right) .
\end{aligned}
$$

Proof: We first re-write the rational function and the logarithm function in terms of Meijer-G function as [30, Eq. 10] and [30, Eq. 11]

$$
\frac{1}{(x+b)^{m}}=\frac{1}{(b)^{m}(m-1) !} G_{1,1}^{1,1}\left(\begin{array}{c|c}
x & 1-m \\
b & 0
\end{array}\right),
$$

and

$$
\log (1+x)=G_{2,2}^{1,2}\left(\begin{array}{ll}
x & 1,1 \\
1,0
\end{array}\right) .
$$

By substituting (24) and (25) into (22), we can obtain the desired result with the help of [31].

Having established the result in Proposition 2, we are now in a position to derive the system ergodic capacity, which is given in Lemma 3.

Lemma 3 The exact closed-form expression of the system ergodic capacity for cognitive underlay SIMO networks over equally correlated Rayleigh fading channels is given as

$$
\begin{aligned}
C= & \alpha \sum_{n=1}^{\mathcal{N}} \sum_{k_{1}, k_{2}, \ldots, k_{n}=0}^{\infty} \bar{\sum}\left(\begin{array}{c}
\mathcal{N} \\
n
\end{array}\right) \\
& \times\left[\frac{(-1)^{n}\left(\sum_{i=1}^{n} k_{i}\right) !\left(\sum_{i=1}^{n} m_{i}\right) !\left(\beta_{1, n}\right)^{1+\sum_{i=1}^{n} k_{i}}}{\prod_{i=1}^{n}\left[k_{i} ! m_{i} !\right](n)^{1+\sum_{i=1}^{n} m_{i}}}\right] \mathcal{W},
\end{aligned}
$$

where $\mathcal{W}$ is given by

$$
\begin{aligned}
\mathcal{W}= & \left(\sum_{i=1}^{n} m_{i}\right) J_{2}\left(\beta_{2, n}, \sum_{i=1}^{n} m_{i}, \sum_{i=1}^{n} m_{i}+1\right) \\
& -\left(\sum_{i=1}^{n} m_{i}+1\right) J_{2}\left(\beta_{2, n}, \sum_{i=1}^{n} m_{i}+1, \sum_{i=1}^{n} m_{i}+2\right) .
\end{aligned}
$$

Furthermore, $J_{2}$ is defined as

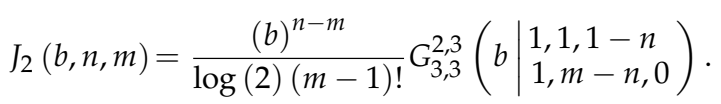

Proof: By substituting (11) into (21) and then making use the result in Lemma 3, we arrive at the desired result, which also completes the proof.

\section{Numerical Results}

In this section, Monte-Carlo simulations are used to confirm the correctness of the analysis results. For illustrative purpose, we consider a 2-D normalized 


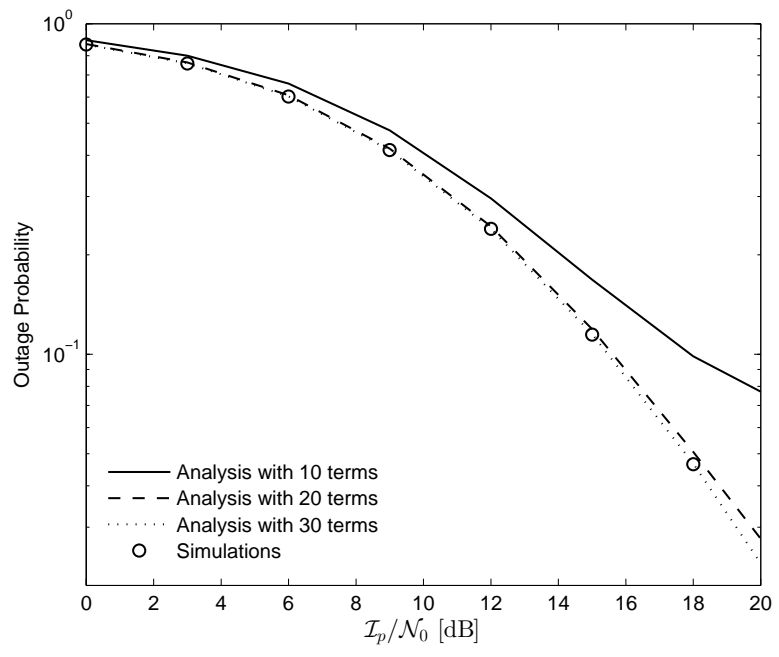

Figure 2. Bounds for outage probability, $\mathcal{N}=2, \rho=0.8$

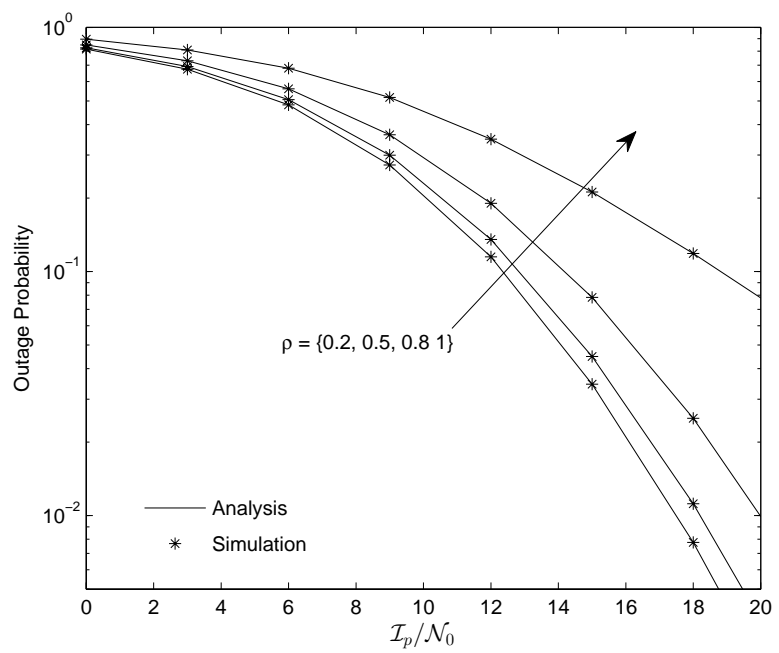

Figure 3. Effect of $\rho$ on outage probability, $\mathcal{N}=3$.

plane, where the secondary transmitter and receiver are placed at coordinates $(0,0)$ and $(1,0)$, respectively, while the primary user is at $(0.5,0.5)$ unless otherwise stated. To take into account the path-loss effect, fading channel gains are modeled as $\lambda=d_{A B}^{-\eta}$, where $d_{A B}$ denotes the distance between node $A \in\{\mathrm{Tx}\}$ and $B \in$ $\{\mathrm{Rx}, \mathrm{P}\}$ and $\eta$ is the path-loss exponent. Without loss of generality, we set the expected rate to $2(R=2 \mathrm{bps} / \mathrm{Hz})$ and the path-loss exponent to $3(\eta=3)$, respectively.

The computation of the outage probability, average bit error rate and ergodic capacity according to (14), (16), and (26) requires the computation of an infinite series. To efficiently compute the series, we derive upper bounds for the performance metrics by truncating the series.

In Figure 2, we plot the outage probability as a function of average SNRs. We consider three cases of truncated terms: 10, 20 and 30. It can be seen that the truncated series will provide a better approximation if more terms in the series are kept. In particular, the case of 30 truncated terms gives a good fit and outperforms the other cases. Hereafter, all of the analysis results will be with 30 truncated terms.

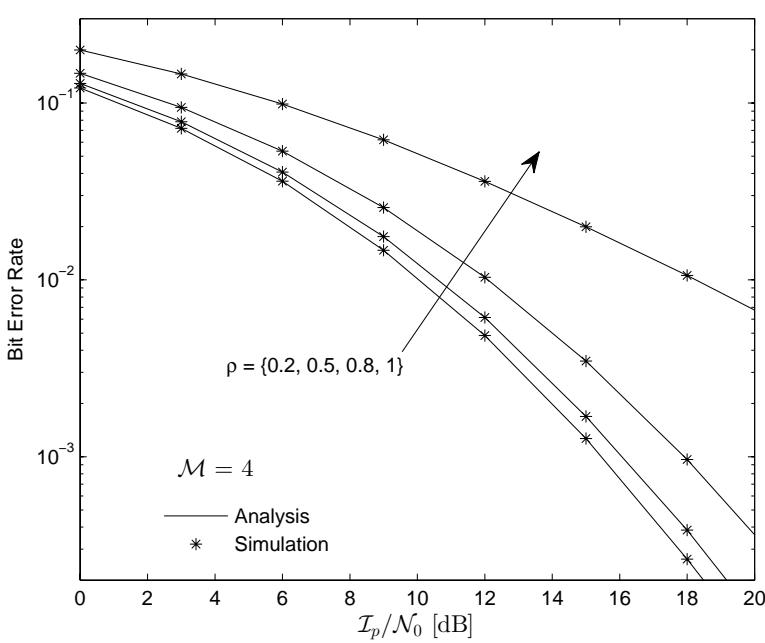

Figure 4. Effect of $\rho$ on average bit error rate, $\mathcal{N}=3$.

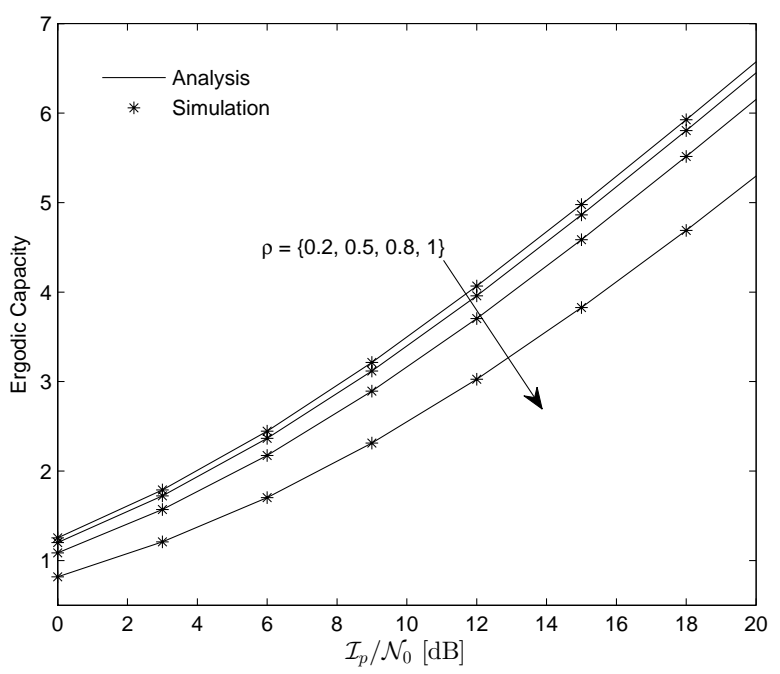

Figure 5. Effect of $\rho$ on ergodic capacity, $\mathcal{N}=3$.

In Figure 3, 4, and 5, we investigate the effect of antenna correlation on the system outage probability, bit error rate and ergodic capacity, respectively. We considered four values of $\rho$ increasing from $0.2,0.5$, 0.8 to 1 . As expected, when $\rho$ increases, the system performance is decreased. It can be seen that the system performance is worst when $\rho$ approaches 1 . It can be explained by making use the fact that all channels from Tx to Rx are fully correlated. We also observe that the system diversity order is a decreasing function of $\rho$. In addition, the analysis results are in excellent agreement with the simulation ones.

In Figure 6, 7, and 8, we study the effect of the primary user's positions on the system performance by varying the $\mathrm{x}$-coordinate while fixing the $\mathrm{y}$-coordinate. As we can see, the system performance improves significantly when the primary user moves far away the secondary transmitter. It is due to the fact that the transmit power is inversely proportional to the interference channel coefficient. Compared with the correlation effect, the primary user position is more significant. For example, with the same $y$-coordinate, the outage probability decreases 10 times when the $\mathrm{x}$-coordinate changes 


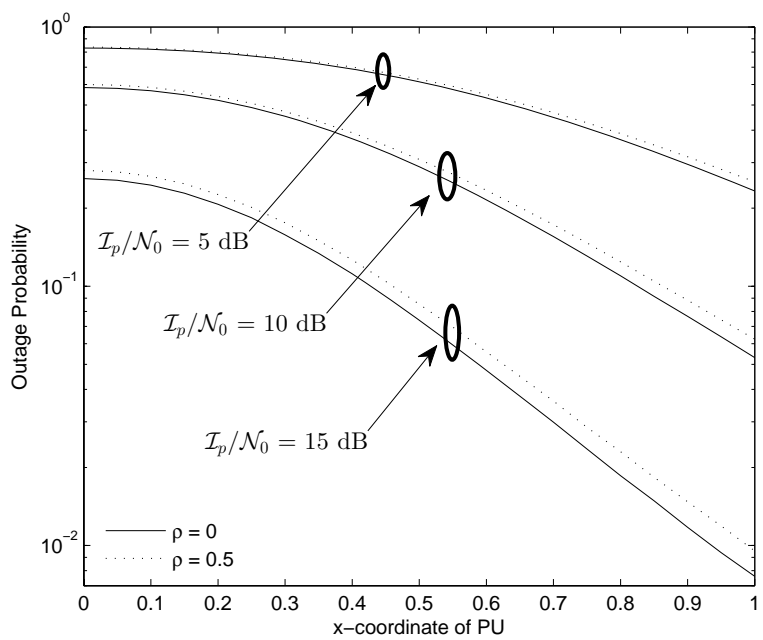

Figure 6. Effect of PU's positions on outage probability, $\mathcal{N}=2$

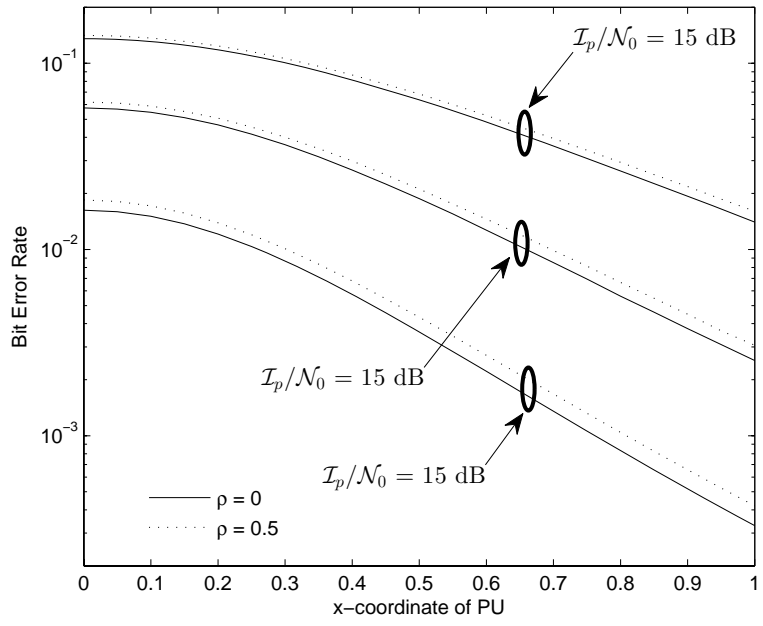

Figure 7. Effect of PU's positions on average bit error rate, $\mathcal{N}=2$

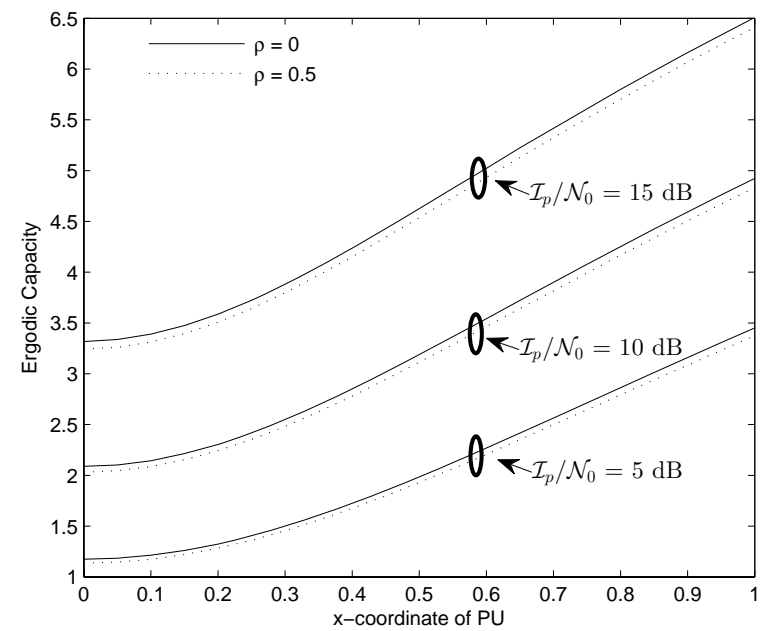

Figure 8. Effect of PU's positions on ergodic capacity, $\mathcal{N}=2$.

from 0.4 to 0.9 . While the system outage probability only gains $1 \mathrm{~dB}$ when $\rho$ increases from 0 to 0.5 .

In Figures 9, 10, and 11, we investigate the effect of the number of receiving antennas on the system performance. For the low SNR regime, the gap performance

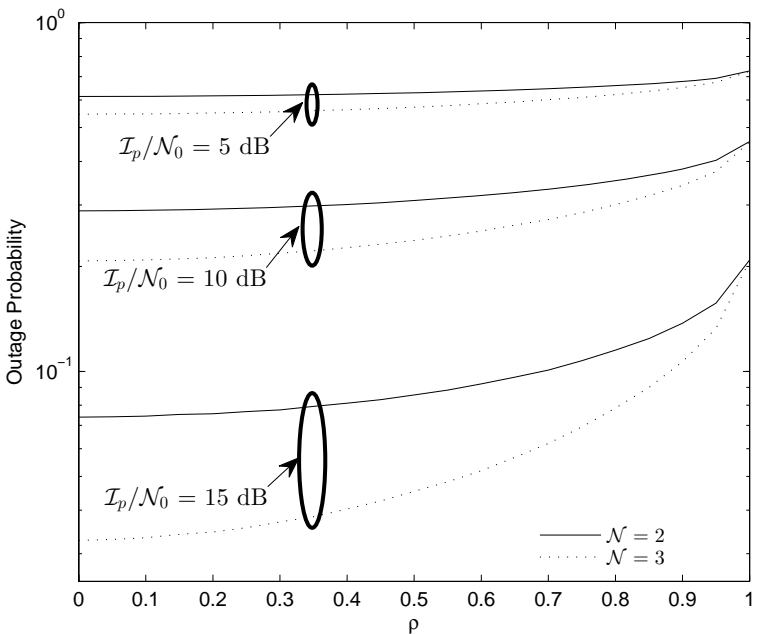

Figure 9. Effect of number of receive antennas on outage probability.

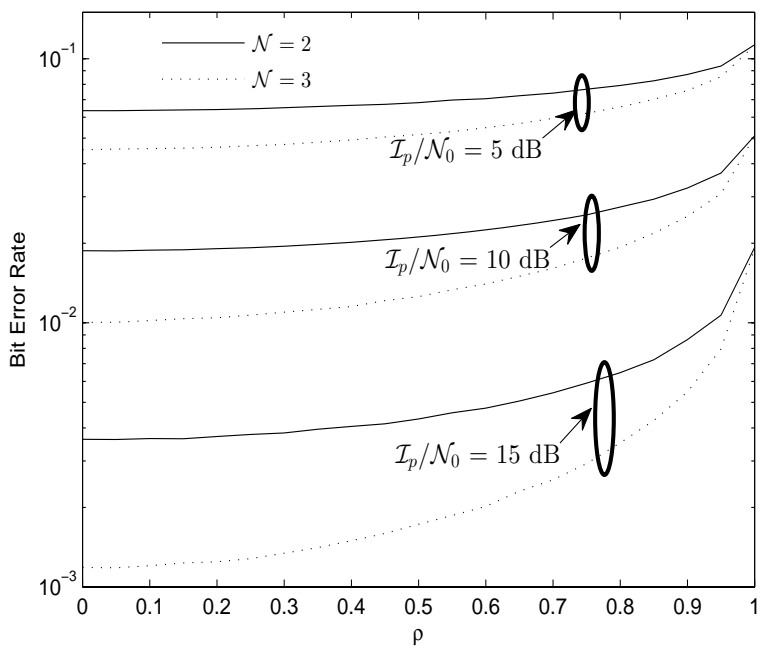

Figure 10. Effect of number of receive antennas on average bit error rate.

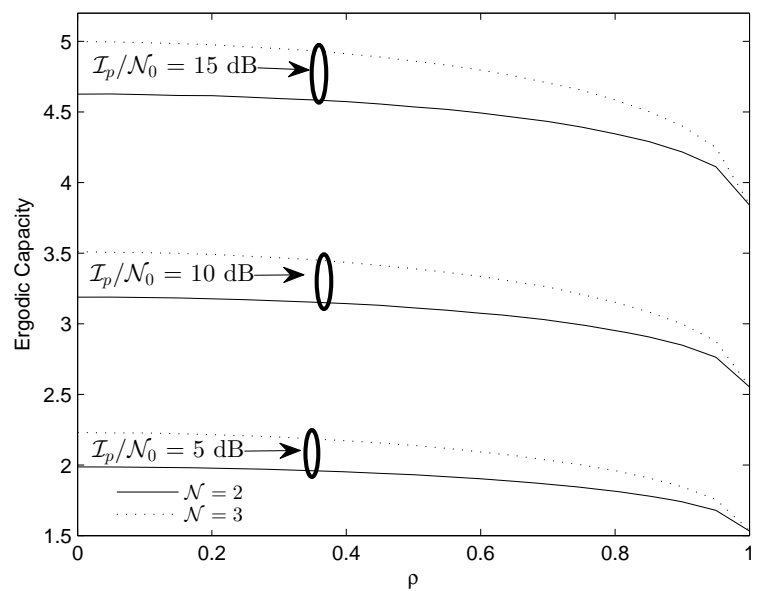

Figure 11. Effect of number of receive antennas on ergodic capacity.

between $N=2$ and $N=3$ is almost constant and does not depend on $\rho$. However, for the large SNR regime, the performance gap is very large at low $\rho$ and very small at $\rho$. The phenomenon are the same not only for outage probability but also for the average bit error rate and system ergodic capacity. 


\section{Conclusion}

In this paper, the performance of cognitive underlay SIMO networks over equally correlated Rayleigh fading channels has been studied. Three performance metrics including outage probability, average bit error rate and ergodic capacity are derived in closed-form in terms of infinite series. To provide a good approximation with efficient computation, we also proposed efficient bounds by truncating series. Numerical results show that the bounds with the first 30 terms are in excellent agreement with the simulation results. In addition, increasing the number of receiving antenna can improve the secondary system performance when both the correlation effect and interference links are taken into account. The primary user's location has significant effect on the performance of the secondary performance. And the correlation effect among antennas limits the system diversity order.

\section{REFERENCES}

[1] S. Haykin, "Cognitive radio: brain-empowered wireless communications," IEEE Journal on Selected Areas in Communications, vol. 23, no. 2, pp. 201-220, Feb. 2005.

[2] I. F. Akyildiz, L. Won-Yeol, M. C. Vuran, and S. Mohanty, "A survey on spectrum management in cognitive radio networks [cognitive radio communications and networks]," IEEE Communications Magazine, vol. 46, no. 4, pp. 40-48, Apr. 2008.

[3] A. Goldsmith, S. A. Jafar, I. Maric, and S. Srinivasa, "Breaking spectrum gridlock with cognitive radios: An information theoretic perspective," Proceedings of the IEEE, vol. 97, no. 5, pp. 894-914, May 2009.

[4] B. Tae Won, C. Wan, J. Bang Chul, and S. Dan Keun, "Multi-user diversity in a spectrum sharing system," IEEE Transactions on Wireless Communications, vol. 8, no. 1, pp. 102-106, Aug. 2009.

[5] Z. Caijun, T. Ratnarajah, and W. Kai-Kit, "Outage analysis of decode-and-forward cognitive dual-hop systems with the interference constraint in Nakagami- $m$ fading channels," IEEE Transactions on Vehicular Technology, vol. 60, no. 6, pp. 2875-2879, Jun. 2011.

[6] V. N. Q. Bao and D. Q. Trung, "Exact outage probability of cognitive underlay DF relay networks with best relay selection," IEICE Transactions on communications, vol. E95B, no. 6, pp. 2169-2173, Jun. 2012.

[7] T. Duong, P. Yeoh, V. N. Q. Bao, M. Elkashlan, and N. Yang, "Cognitive relay networks with multiple primary transceivers under spectrum-sharing," IEEE Signal Processing Letters, vol. 19, no. 11, pp. 741 - 744, Nov. 2012.

[8] Y. Ping, L. Liping, and Q. Jiayin, "Outage performance of cognitive relay networks with interference from primary user," IEEE Communications Letters, vol. 16, no. 10, pp. 1695-1698, Oct. 2012.

[9] H. Jafarkhani, Space-time coding: theory and practice. Cambridge: Cambridge Univ. Press, 2005.

[10] S. Sanayei and A. Nosratinia, "Antenna selection in MIMO systems," IEEE Communications Magazine, vol. 42, no. 10, p. 6873, Oct. 2004.

[11] B. Nguyen, R. Afolabi, and K. Kim, "Dependence of outage probability of cooperative systems with single relay selection on channel correlation," IEEE Communications Letters, vol. 17, no. 11, pp. 2060 - 2063, Nov. 2013.

[12] Sekulovic, x, N., Stefanovic, M., Denic, D., and Aleksic, "Performance analysis of signal-to-interferenceplusnoise ratio-based selection diversity over correlated
Rayleigh fading channels," IET Communications, vol. 5, no. 2, pp. 127-134, Feb. 2011.

[13] T. Sanguanpuak, N. Rajatheva, and A. Taparugssanagorn, "Performance analysis of OSTBC for partial relay selection with correlated antennas over Nakagami-m fading," IEEE Wireless Communications Letters, vol. 2, no. 3, pp. 355-358, Feb. 2013.

[14] G. Hongbo and V. C. M. Leung, "Orthogonal transmissions for spectrum underlay MISO cognitive radio," IEEE Transactions on Wireless Communications, vol. 11, no. 4, pp. 1266-1270, Apr. 2012.

[15] C. Nguyen Van, V. N. Q. Bao, and N. Nguyen Luong, "On the performance of cognitive underlay Alamouti space-time coding schemes," in Proceedings of the International Conference on Advanced Technologies for Communications (ATC'13), Ho Chi Minh City, Vietnam, 2013, pp. 23-27.

[16] R. Sarvendranath and N. B. Mehta, "Antenna selection in interference-constrained underlay cognitive radios: SEP-optimal rule and performance benchmarking," IEEE Transactions on Communications, vol. 61, no. 2, pp. 496506, Feb. 2013.

[17] S. Raza Zaidi, M. Ghogho, D. McLernon, and A. Swami, "Achievable spatial throughput in multi-antenna cognitive underlay networks with multi-hop relaying," IEEE Journal on Selected Areas in Communications, vol. 31, no. 8, pp. 1543- 1558, Aug. 2013.

[18] T. Tu Lam, V. N. Q. Bao, and N. Pham Thi Dan, "Outage performance of underlay SIMO networks over equally correlated Rayleigh fading channels," in The First NAFOSTED Conf. Inf. and Computer Science, Hanoi, Vietnam, pp. 63-72.

[19] C. Yunxia and C. Tellambura, "Performance analysis of $l$-branch equal gain combiners in equally correlated Rayleigh fading channels," Communications Letters, vol. 8, no. 3, pp. 150-152, Aug. 2004

[20] — " "Distribution functions of selection combiner output in equally correlated Rayleigh, Rician, and Nakagami- $m$ fading channels," IEEE Transactions on Communications, vol. 52, no. 11, pp. 1948-1956, Nov. 2004.

[21] M. R. Bhatnagar, "On the capacity of decode-andforward relaying over Rician fading channels," IEEE Communications Letters, vol. 17, no. 6, pp. 1100-1103, Jun. 2013.

[22] A. H. Nuttall, "Some integrals involving the $Q$-function," DTIC Document, Report, 1972.

[23] T. Q. Duong, V. N. Q. Bao, and H.-J. Zepernick, "Exact outage probability of cognitive AF relaying with underlay spectrum sharing," Electronics letters, pp. 1001-1002, Aug. 2011.

[24] V. N. Q. Bao, H. Y. Kong, and S. W. Hong, "Performance analysis of $M$-PAM and M-QAM with selection combining in independent but non-identically distributed rayleigh fading paths," in Proceedings of the IEEE 68th Vehicular Technology Conference (VTC'08-Fall), 2008, Conference Proceedings, pp. 1-5.

[25] O. Olabiyi and A. Annamalai, "New series representations for generalized Nuttall Q-function with applications," in Proceedings of the IEEE Consumer Communications and Networking Conference (CCNC'12), Las Vegas, NV, 2012, pp. 782-786.

[26] I. S. Gradshteyn, I. M. Ryzhik, A. Jeffrey, and D. Zwillinger, Table of integrals, series and products, 7th ed. Amsterdam ; Boston: Elsevier, 2007.

[27] K. Cho and D. Yoon, "On the general BER expression of one- and two-dimensional amplitude modulations," IEEE Transactions on Communications, vol. 50, no. 7, pp. 1074-1080, Jul. 2002.

[28] [Online]. Available: http://functions.wolfram.com/06. 27.26.0006.01

[29] [Online]. Available: http://functions.wolfram.com/07. 34.21.0013.01

[30] V. Adamchik and O. Marichev, "The algorithm for calcu- 
lating integrals of hypergeometric type functions and its realization in reduce system," in Proceedings of the international symposium on Symbolic and algebraic computation. ACM, pp. 212-224.

[31] [Online]. Available: http://functions.wolfram.com/07. 34.21.0086.01

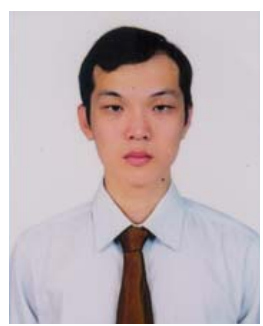

Thanh Tu-Lam received the B.E. and the M.S. degrees in Electronics and Telecommunications Engineering from Ho Chi Minh City University of Technology and Posts and Telecommunications Institute of Technology (PTIT), Vietnam in 2009 and 2013, respectively. Currently, he is an assistant researcher at Wireless Communications Laboratory of PTIT. His major research interests are cooperative communications, diversity technique, physical layer security, cognitive radio, stochastic geometry and energy harvesting.

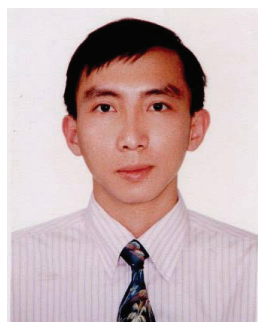

Vo Nguyen Quoc Bao was born in Nha Trang, Khanh Hoa Province, Vietnam. He received the B.E. and M.Eng. degree in electrical engineering from Ho Chi Minh City University of Technology (HCMUT), Vietnam, in 2002 and 2005, respectively, and Ph.D. degree in electrical engineering from University of Ulsan, South Korea, in 2009. In 2002, he joined the Department of Electrical Engineering, Posts and Telecommunications Institute of Technology (PTIT), as a lecturer. Since February 2010, he has been with the Department of Telecommunications, PTIT, where he is currently an Assistant Professor. His major research interests are modulation and coding techniques, MIMO systems, combining techniques, cooperative communications, and cognitive radio. Dr. Bao is a member of Korea Information and Communications Society (KICS), The Institute of Electronics, Information and Communication Engineers (IEICE) and The Institute of Electrical and Electronics Engineers (IEEE). He is currently serving as the Editor of Transactions on Emerging Telecommunications Technologies (Wiley ETT). He is also a Guest Editor of EURASIP Journal on Wireless Communications and Networking, special issue on "Cooperative Cognitive Networks" and IET Communications, special issue on "Secure Physical Layer Communications".

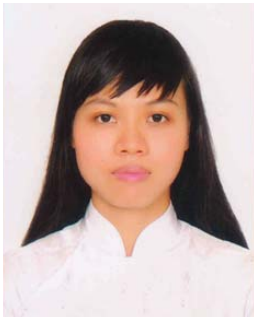

Pham Thi Dan Ngoc received the B.E. and the M.S. degrees in Electronics and Telecommunications Engineering from Posts and Telecommunications Institute of Technology (PTIT), Vietnam in 2009 and 2013, respectively. Currently, she is an assistant researcher at Wireless Communications Laboratory of PTIT. Her major research interests are cooperative communications, diversity technique, physical layer security, cognitive radio and energy harvesting.

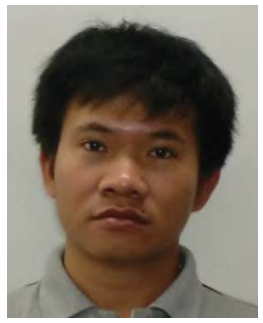

Tran Trung Duy received the B.E. degree in Electronics and Telecommunications Engineering from Ho Chi Minh City University of Technology, Vietnam, in 2007. In 2013, he received the PhD degree in electrical engineering from University of Ulsan, South Korea. He is currently a lecturer in the Department of Electrical Engineering of Posts and Telecommunications Institute of Technology (PTIT). $\mathrm{He}$ is also a senior researcher at Wireless Communication Laboratory of PTIT. His major research interests are cooperative communications, cognitive radio, and physical layer security. 